\title{
CORRELATION BETWEEN NO-SLIP AND SLIP BOUNDARY CONDITIONS ASSOCIATED WITH A TWO-DIMENSIONAL NAVIER-STOKES FLOWS IN A PLANE DIFFUSER
}

\author{
RANIS IBRAGIMOV $^{1}$ and VESSELIN VATCHEV ${ }^{2}$ \\ ${ }^{1}$ Department of Mathematics \\ Wenatchee Valley College \\ 1300 Fifth St. \\ Wenatchee, WA 98801 \\ U. S. A. \\ e-mail: ribragimov@wvc.edu \\ 2Department of Mathematics \\ University of Texas Rio Grande Valley \\ One West Boulevard \\ Brownsville, TX 78520 \\ U. S. A. \\ e-mail: vesselin.vatchev@utrgv.edu
}

2020 Mathematics Subject Classification: 35J05, 76D05, 76N10, 76D07.

Keywords and phrases: Navier-Stokes flows, plane diffuser, effects of viscosity, slip boundary conditions.

Received March 4, 2021

(C) 2021 Scientific Advances Publishers

This work is licensed under the Creative Commons Attribution International License (CC BY 3.0).

http://creativecommons.org/licenses/by/3.0/deed.en US

Open Access (cc) (1)




\begin{abstract}
We examine the viscous effects of slip boundary conditions for the model describing two-dimensional Navier-Stokes flows in a plane diffuser. It is shown that the velocity profile is related to a half period shifted Weierstrass function with two parameters. This allows to approximate the explicit solution by a Taylor series expansion with two new micro- parameters, that can be measured in physical experiments. It is shown that the assumption for no-slip boundary conditions is stable in the sense that a small perturbation of the boundary values result in a small perturbation in the solutions.
\end{abstract}

\title{
1. Introduction
}

The governing equations, along with the appropriate constitutive relations describe fully the fluid flow within a given geometry. However, mathematical model cannot be solved unless we specify the boundary conditions for the problem. Physically, the need for boundary conditions is not surprising, because the boundary conditions provide information on the way the fluid interacts with its surroundings. Mathematically, this is expected because upon integration, the differential equations yield constants that are fixed using boundary conditions.

In this paper, we will consider the viscous effects of slip boundary conditions for the model describing two-dimensional Navier-Stokes flows in a plane diffuser. A diffuser is an engineering device used within internal flow systems widely used in industry. For example, ejectors used in refrigeration systems as entrainment and compression components or expanders, attract interest from engineeries as a means of more efficient energy use $([1,6,19])$. Commonly, such comples flows in a diffuser are encountered in turbomachines between a compressor and a combustor or at the exit of a turbine, as shown in Figure 1. Diffusing passages may also be encountered in small-scale devices such as fluidic actuators and MicroElectro-Mechanical Systems. 


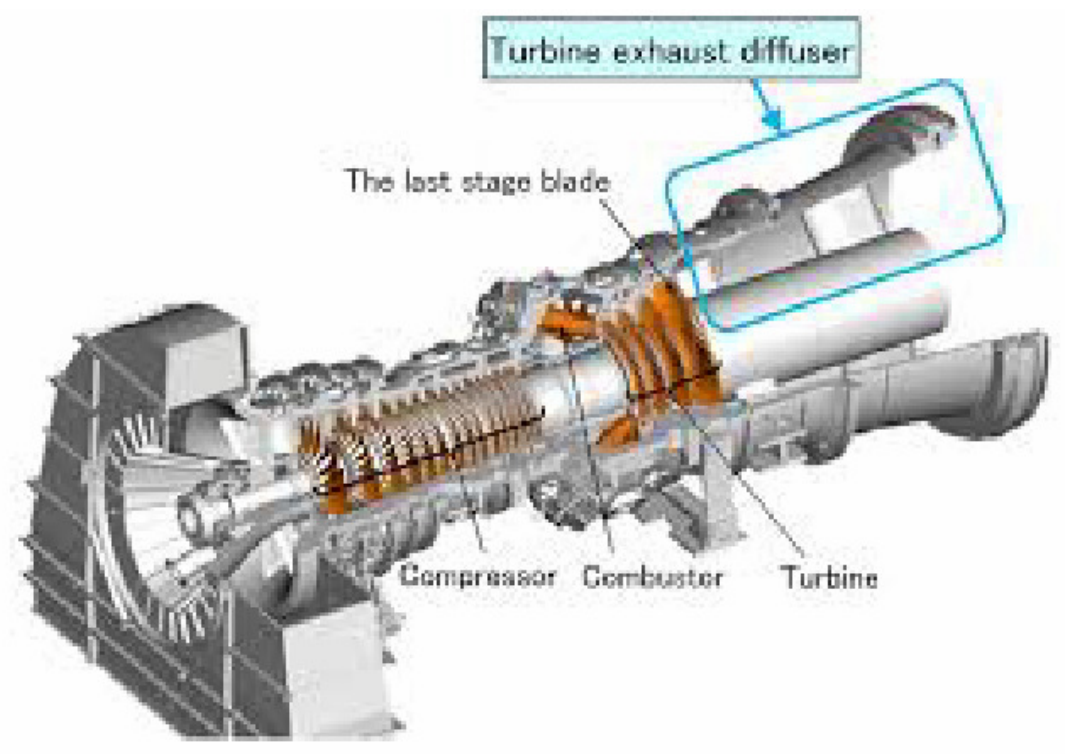

Figure 1. Turbine exhaust diffuser. Sourse [10].

It is known that in nozzles the static pressure decreases in the flow direction (favourable pressure gradient) [3, 11]. However, fluid particle .owing through a diffuser experiences an increasing static pressure (adverse pressure gradient) resulting to complex fluid phenomena, including flow separation and unsteadiness to transitory stall and violent flow-excited static pressure fluctuations ([4,20]). Such flows are hard to predict quantitatively, although they are qualitatively understood $[5,18]$.

In the vast majority of problems, we have fluid flowing along solid boundaries. Consistent with continuum theory, the no-slip boundary condition states that there is no relative motion between fluid particles and the solid boundaries with which they are in contact. That is,

$$
V_{\text {fluid }}=V_{\text {wall }} \text {. }
$$


No slip condition is the reason for formation of boundary layer in a fluid flow [13]. If the wall is not moving, then the fluid velocity at the wall is

$$
V_{\text {fluid }}=0 .
$$

Basically the molecules of the fluid crash into the molecules of the wall and get stopped. Then they dribble out and get crashed into by faster molecules farther out in the flow.

For example, all gases experience some slip at the wall where typically the slip velocity $V$ (difference between tangential velocities of wall and gas) is given by a condition like

$$
V=-a_{1} s_{t n}-a_{2} q_{t}
$$

where $s_{t n}$ is the tangential-normal element of pressure tensor, $q_{t}$ is tangential heat flux, and $a_{1}, a_{2}$ are coefficients that depend on the state of the gas. The first term describes classical slip, the second term describes what is known as thermal transpiration: a flow driven by a temperature gradient along the wall ([16]).

Despite its apparent simplicity, the no-slip boundary condition cannot be derived from first principles and could, in theory, be violated. In addition, it leads to some physical inconsistencies, that are not resolved completely. For instance, the no-slip boundary condition cannot explain the motion of a liquid interface in contact with a solid boundaries; according to this condition, the liquid interface in a partially filled glass must remain stationary with respect to the glass when the glass is moved. At the microscopic level at least, we know that this is not the case. Consider also the flow near an exist of a pipe. According to the no-slip condition, the velocity of fluid particles near the exit is mathematically singular as the velocity changes from zero to a finite value. 
It has been shown in [15] that the physical inconsistencies can be removed mathematically by assuming that the fluid particles in contact with solid surface slip. This means that fluid particles in contact with the wall can slide, resulting in an apparent fluid velocity. As a matter of fact, there is a strong evidence (see, e.g., $[14,17])$ that this occurs in many nonNewtonian fluids. In such cases, the slip velocity $V_{s}$ is assumed to be proportional to the shear along the wall,

$$
V_{s}=\lambda \tau_{w},
$$

where $\lambda$ is a proportionality constant to be determined experimentally and $\tau_{w}$ is shear along the wall (see also [9]). However, for the most Newtonian fluids there are not many experimental evidence to support the presence of a slip [12]. In the present work, we consider a special case of a diffuser for which we study the relation between $V_{s}$ and the shear. We show that no-slip boundary conditions can be considered as a limit case, $\lambda \rightarrow 0$, of the slip boundary conditions and we study the effect on the flow for different ranges of $\lambda$.

\section{Mathematical Model and Problem Geometry}

In two dimensions, we introduce polar coordinates $x=r \cos \theta$, $y=r \sin \theta$ and write two-dimensional Navier-Stokes equations describing a fully developed flow within a plane diffuser, as a two dimensional channel with the line source at the origin, as shown in Figure 2 


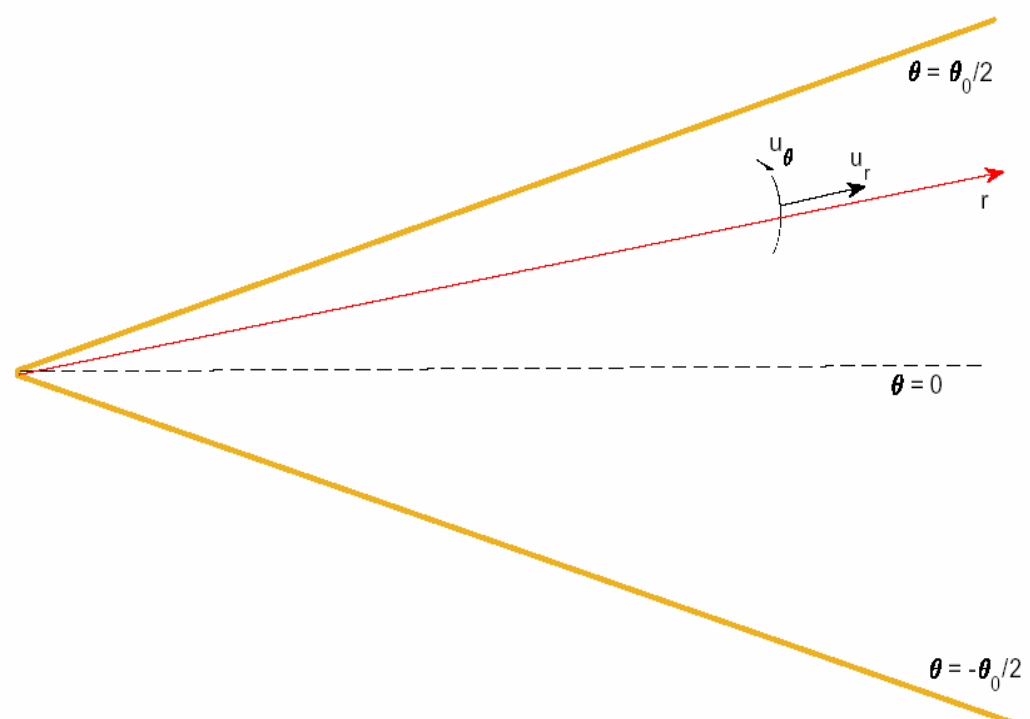

Figure 2. Problem geometry.

$$
\begin{gathered}
u_{r} \frac{\partial u_{r}}{\partial r}+\frac{u_{\theta}}{r} \frac{\partial u_{r}}{\partial \theta}-\frac{u_{\theta}^{2}}{r}=-\frac{1}{\rho} \frac{\partial p}{\partial r}+v\left(\nabla^{2} u_{r}-\frac{2}{r^{2}} \frac{\partial u_{\theta}}{\partial \theta}-\frac{u_{r}}{r^{2}}\right) \\
u_{r} \frac{\partial u_{\theta}}{\partial r}+\frac{u_{\theta}}{r} \frac{\partial u_{\theta}}{\partial \theta}+\frac{u_{\theta} u_{r}}{r}=-\frac{1}{\rho} \frac{1}{r} \frac{\partial p}{\partial \theta}+v\left(\nabla^{2} u_{\theta}+\frac{2}{r^{2}} \frac{\partial u_{r}}{\partial \theta}-\frac{u_{\theta}}{r^{2}}\right) \\
\frac{\partial}{\partial r}\left(r u_{r}\right)+\frac{\partial u_{\theta}}{\partial \theta}=0
\end{gathered}
$$

where $u_{r}$ and $u_{\theta}$ are the radial and angular components of the velocity vector, $\rho$ is a constant density, $p(r, \theta)$ is a pressure and

$$
\nabla^{2}=\frac{\partial^{2}}{\partial r^{2}}+\frac{1}{r} \frac{\partial}{\partial r}+\frac{1}{r^{2}} \frac{\partial^{2}}{\partial \theta^{2}}
$$

From (7), we can introduce the stream function $\vartheta(r, \theta)$ via

$$
u_{r}=\frac{1}{r} \frac{\partial \psi}{\partial \theta}, \quad u_{\theta}=-\frac{\partial \psi}{\partial r}
$$




\section{Basic Flow using Stream Function}

The basic flow is assumed to be purely radial emerging from a line source, so we can look for particular solution for the problem in the form

$$
u_{r}^{0}=u_{r}^{0}(r, \theta), \quad u_{\theta}^{0}=0, \quad p^{0}=p^{0}(r, \theta) .
$$

Then, as follows from Equation (5)-(6), the basic flow (10) satisfies

$$
\begin{gathered}
u_{r}^{0} \frac{\partial u_{r}^{0}}{\partial r}=-\frac{1}{\rho} \frac{\partial p^{0}}{\partial r}+v\left(\frac{\partial^{2} u_{r}^{0}}{\partial r^{2}}+\frac{1}{r} \frac{\partial u_{r}^{0}}{\partial r}+\frac{1}{r^{2}} \frac{\partial^{2} u_{r}^{0}}{\partial \theta^{2}}-\frac{u_{r}^{0}}{r^{2}}\right) \\
0=-\frac{1}{\rho} \frac{1}{r} \frac{\partial p^{0}}{\partial \theta}+v\left(\frac{2}{r^{2}} \frac{\partial u_{r}^{0}}{\partial \theta}\right)
\end{gathered}
$$

Also, it follows from (7) that $\chi$ is a function of $\theta$ only.

Hereafter the index zero is omitted. We next write the model (11)-(12) in terms of $x$ as follows:

$$
\begin{gathered}
\frac{1}{r^{3}}\left(\frac{\partial \psi}{\partial \theta}\right)^{2}=\frac{1}{\rho} \frac{\partial p}{\partial r}-v\left(\frac{1}{r^{3}} \frac{\partial^{3} \psi}{\partial \theta^{3}}\right) \\
0=-\frac{1}{p r} \frac{\partial p}{\partial \theta}+v\left(\frac{2}{r^{3}} \frac{\partial^{2} \psi}{\partial \theta^{2}}\right)
\end{gathered}
$$

We note that the pressure $p$ can be determined from Equation (14) as

$$
\frac{1}{\rho} p(\theta, r)=\frac{2 v}{r^{2}} \frac{\partial \psi}{\partial \theta}+\alpha(r),
$$

where $\alpha$ is an arbitrary function.

\section{Derivation of the Main Equation}

We rewrite the Equation (13), in which $p$ is given by (15)

$$
\frac{\partial^{3} \not}{\partial \theta^{3}}+4 \frac{\partial p}{\partial \theta}+\frac{1}{v}\left(\frac{\partial p}{\partial \theta}\right)^{2}=\frac{r^{3}}{v} \frac{d \alpha}{d r} .
$$


The left part of (16) depends only on $\theta$ whereas the right side depends only on $r$. Hence, the both sides are equal to a constant, say $c_{1}$. Then the right side of (16) yields

$$
\alpha(r)=-\frac{c_{1} v}{2 r^{2}}+\frac{p_{0}}{\rho},
$$

where $p_{0}=$ const. By substituting $\alpha(r)$ given by (17) into (15), we obtain the following expression for the pressure:

$$
p(\theta, r)=\frac{v \rho}{r^{2}}\left[2 \frac{\partial p}{\partial \theta}-\frac{c_{1}}{2 \rho}\right]+p_{0}
$$

Next we modify the main equation and the boundary conditions. We denote

$$
U(\theta)=\frac{\partial \psi}{\partial \theta}
$$

Then in terms of $U$, the Equation (16) is written as

$$
\frac{d^{2} U}{d \theta^{2}}+4 U+\frac{1}{v} U^{2}=c_{1}
$$

where $c_{1}$ is an arbitrary constant. Multiplying (20) by $d U$ and integrating we get the first order equation

$$
\left(\frac{d U}{d \theta}\right)^{2}=-\frac{2}{3 v} U^{3}-4 U^{2}+2 c_{1} U-2 c_{2},
$$

where $c_{2}$ is a new constant.

We note that the variables in Equation (21) can be separated, which provides us with the relation between $U$ and $\theta$ in the form

$$
\theta=\int_{U_{1}}^{U} \frac{d \xi}{ \pm \sqrt{f(\xi)}}
$$


where

$$
f(\xi)=-\frac{2}{3 v} \xi^{3}-4 \xi^{2}+2 c_{1} \xi-2 c_{2}
$$

The form (22) suggests that we can find the exact solution of the general equation (21) by the substitution

$$
U(\theta)=-6 v\left(\frac{1}{3}+y(\theta)\right)
$$

where $y$ is solution of the equation

$$
y^{\prime \prime}=6 y^{2}-\frac{g_{2}}{2}
$$

for a real constant $g_{2}$. The general solution of the above equation is the Weierstrass elliptic $P$ function, denoted $\wp$, [7]. The function $\wp\left(\theta, g_{2}, g_{3}\right)$ is a well known function that posses interesting properties and is the solution for our BVP problem. The first parameter $g_{2}$ appears in Equation (25) whereas the second parameter $g_{3}$ appears after integration of Equation (25). These two parameters $g_{2}, g_{3}$ has to be determined by the boundary conditions. Next we provide brief overview of the function $\wp$ and it's application to our model.

\section{Application of the Weierstrass Elliptic Function}

The Weierstrass elliptic function $\wp$ is well studied and has many applications in different branches of pure and applied mathematics, a good source for reference is [7]. The function $\wp$ is doubly periodic with complex periods $w_{1}, w_{2}$ and can be defined as the solution of the equation

$$
\wp^{\prime \prime}=6 \wp^{2}-\frac{g_{2}}{2}
$$


or the equivalent first order DE

$$
\wp^{2}=4 \wp^{3}(z)-g_{2} \wp(z)-g_{3},
$$

where $g_{2}$ and $g_{3}$ are real valued constants.

The function $\wp$ is even, $\wp(-z)=\wp(z)$, with poles at a lattice generated by the periods including $z=0$ and it has a Laurent expansion near the pole $z=0$ of the form

$$
\wp(z)=\frac{1}{z^{2}}+\frac{g_{2}}{20} z^{2}+\frac{g_{3}}{28} z^{4}+\sum_{k=4}^{\infty} c_{k} z^{2 k}
$$

where

$$
c_{k}=\frac{3}{(2 k+1)(k-3)} \sum_{m=2}^{k-2} c_{m} c_{k-m}
$$

with $c_{2}=\frac{g_{2}}{20}$ and $c_{3}=\frac{g_{3}}{28}$.

We are looking for a real valued function $U$ and hence real valued $\wp(\theta)$ for real $\theta$. From [8] for $w_{1}, w_{2}$ real and hence $g_{2}, g_{3}$ are also real, the general solution of the equation

$$
y^{\prime 2}=4 y^{3}-g_{2} y-g_{3}
$$

is real valued function and it is either $\wp(\theta)$ or $\wp\left(\theta+\frac{w_{1}}{2}\right)$, where $w_{1}$ is one of the periods for the $\wp$ function. We will show that the Equation (20) for the diffuser is solved by the second function for $\theta \in\left[-\frac{\theta_{0}}{2}, \frac{\theta_{0}}{2}\right]$ thus avoiding the poles of $\wp(\theta)$. If $\theta_{0}<2 \min \left(w_{1}, w_{2}\right)$ the function $\wp\left(\theta+\frac{w_{1}}{2}\right)$ has a single minimum and for $\theta$ away from the poles, it is analytic in $\theta, g_{2}, g_{3}$, which provides continuity of the solution with 
respect to the boundary conditions. Small variations from zero initial conditions result in small variations in $U$ so this might justify the zero conditions in some cases. On the other hand since $\wp$ is defined beyond the limits of the interval then (27) provides the relation

$$
\frac{1}{36 v^{2}} U^{\prime 2}\left(\frac{\theta_{0}}{2}\right)=-4\left(\frac{U_{0}}{6 v}+\frac{1}{3}\right)^{3}+g_{2}\left(\frac{U_{0}}{6 v}+\frac{1}{3}\right)-g_{3}
$$

Denoting by $U_{0}$ the boundary value $U\left(\frac{\theta_{0}}{2}\right)$ we see that zero initial condition, i.e., $U_{0}=0$ corresponds to (31) as $U_{0}^{\prime}=36 v^{2}\left(\frac{4}{27}-\frac{g_{2}}{3}-g_{3}\right)$ and is zero only if $\frac{4}{27}-\frac{g_{2}}{3}-g_{3}=0$ but then $U \leq 0$ and is not a feasible solution. We can conclude that $U_{0}=\mu \lambda U_{0}^{\prime}$ is not possible for $U_{0}=0$ unless $\mu=0$. It is interesting to notice that if $U_{0}>0$ than the cubic relation (31) provides a nonlinear relation between the initial values of $U$ and $U^{\prime}$ which is similar in nature to (4). We discuss that fact in Section 8.

The amount of fluid for any $r$ is conserved which results in the conserving condition

$$
\int_{-\frac{\theta_{0}}{2}}^{\frac{\theta_{0}}{2}} U(\theta) d \theta=2 \int_{0}^{\frac{\theta_{0}}{2}} U(\theta) d \theta
$$

It can be expressed in terms of Weierstrass $\zeta$ function, $\frac{d \zeta}{d \theta}=-\wp(\theta)$. The Weierstrass elliptic functions are implemented in Mathematica and we provide an example. 


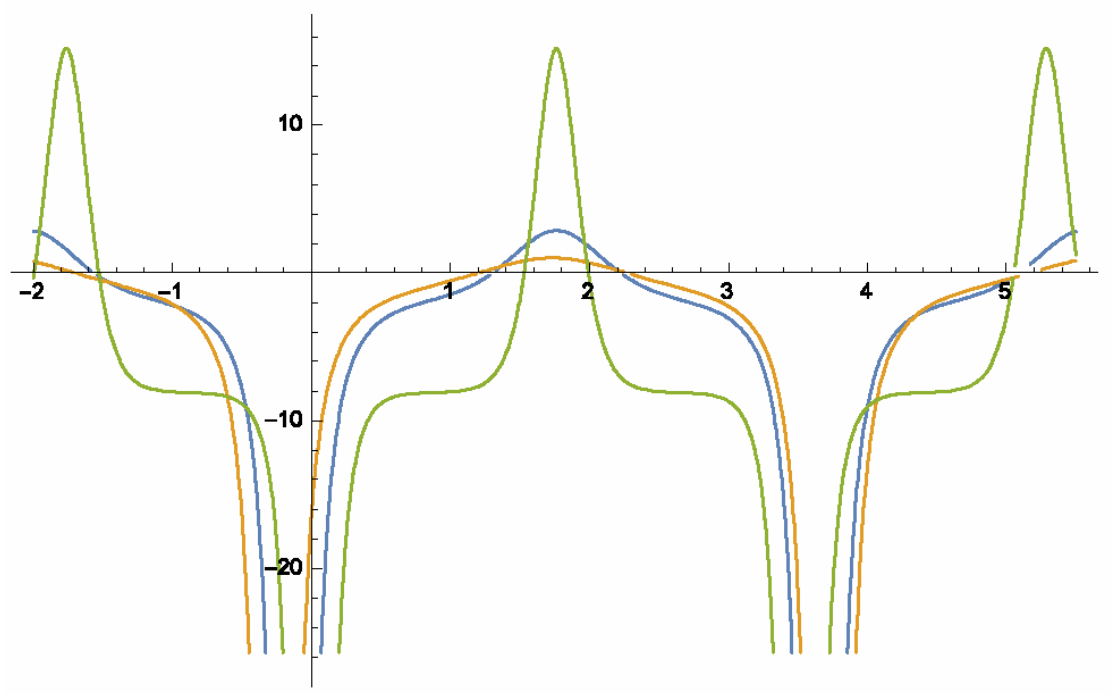

Figure 3. Three graphs for $\wp$ shifted to have the maximum coinciding.

Figure 3 shows three graphs for $\wp$ shifted to have the maximum coinciding. These three graphs represent

$$
\begin{aligned}
& -\wp(x+0.13,29,-37)-1 / 3, \\
& -\wp(x+0.25,2,-7)-1 / 3, \\
& -\wp(x, 724.1,-3750)-1 / 3 .
\end{aligned}
$$

All of the functions are analytic in a neighbourhood around the maximum value but have irremovable singularities outside finite intervals centered at the maximum points. Another observation is that the profile of $\wp$ depending on the parameters $g_{2}, g_{3}$ can vary a lot with the variation of the parameters. In the next section we conduct numerical study of the solutions of the BVP. 


\section{Numerical Study}

The Weierstrass function $\wp\left(\theta, g_{2}, g_{3}\right)$ has different representations. Computationally, the elliptic theta functions provide better convergence than the Laurent series but for our problem we take a different approach. The parameterization by $g_{2}, g_{3}$ is not appropriate for accessing values of the function. The Laurent series expansion is slowly convergent near the poles but using the fact that the function is analytic on the domains of our interest we mimic the construction of $\wp$ for the diffuser problem. We consider two macro-parameters that could be easily measured during an experiment, the maximum velocity of the flow, $E$, and since the amount of fluid is con-served for any $r$ we consider it as a parameter, say $Q$. The following problem is discussed next.

Problem. Approximate the solutions of

$$
u^{\prime \prime}(\theta)=-\frac{1}{v} u^{2}(\theta)-4 u(\theta)+s
$$

with the constrain

$$
\int_{-\frac{\theta_{0}}{2}}^{\frac{\theta_{0}}{2}} u(\theta) d \theta=Q>0,
$$

where $v$ is the viscosity of the fluid, $\theta_{0}, Q$ are positive constants, and $s$ is a real parameter.

The solution of the $\mathrm{DE}(33)$ is $\wp\left(\theta+\frac{w_{1}}{2}\right)$, which is analytic and even function on $\left[-\frac{\theta_{0}}{2}, \frac{\theta_{0}}{2}\right]$, hence it has the Maclaurin series expansion

$$
u(\theta)=\sum_{k=0}^{\infty} a_{k} \theta^{2 k},
$$


with coefficients

$$
a_{k}=\frac{u^{(2 k)}(0)}{(2 k) !}
$$

We introduce two new parameters: the maximum value $U(0)=E$ and $s$ from (33). Using the $\mathrm{DE}$ we can find a recurrence relation for $a_{k}$. For $k=0$ we have $a_{0}=u(0)=E$, for $k=1$ from the equation

$$
a_{1}=\frac{u^{\prime \prime}(0)}{2}=-\frac{1}{2 v} E^{2}-2 E+\frac{s}{2},
$$

and for $k>1$ by differentiating the DE $(2 k-2)$-times we get

$$
\begin{aligned}
\frac{u^{(2 k)}}{(2 k) !} & =-\frac{1}{v(2 k) !}\left(u^{2}\right)^{(2 k-2)}-4 \frac{u^{(2 k-2)}}{(2 k) !} \\
& =-\frac{1}{v(2 k) !} \sum_{m=0}^{2 k-2}\left(\begin{array}{c}
2 k-2 \\
m
\end{array}\right) u^{(2 k-2-m)} u^{(m)}-4 \frac{u^{(2 k-2)}}{(2 k) !}
\end{aligned}
$$

Since $u^{(2 m-1)}(0)=0$ we have

$$
\begin{aligned}
\frac{u^{(2 k)}(0)}{(2 k) !}= & -\frac{1}{v(2 k)(2 k-1)} \sum_{m=0}^{k-1} \frac{u^{(2 k-2-2 m)}(0)}{(2 k-2-2 m) !} \frac{u^{(2 m)}(0)}{(2 m) !} \\
& -\frac{4}{2 k(2 k-1)} \frac{u^{(2 k-2)}(0)}{(2 k-2) !}
\end{aligned}
$$

and

$$
a_{k}=-\frac{1}{2 k(2 k-1)}\left(\frac{1}{v} \sum_{m=0}^{k-1} a_{k-m-1} a_{m}+4 a_{k-1}\right)
$$


The first seven terms are computed as follows:

$$
\begin{aligned}
& a_{0}=E \\
& a_{1}=-\frac{1}{2 v} E^{2}-2 E+\frac{s}{2}, \\
& a_{2}=\frac{-1}{12}\left(\frac{1}{v} 2 a_{0} a_{1}+4 a_{1}\right), \\
& a_{3}=\frac{-1}{30}\left(\frac{1}{v}\left(2 a_{0} a_{2}+a_{1}^{2}\right)+4 a_{2}\right), \\
& a_{4}=\frac{-1}{56}\left(\frac{1}{v}\left(2 a_{0} a_{3}+2 a_{2} a_{1}\right)+4 a_{3}\right), \\
& a_{5}=\frac{-1}{90}\left(\frac{1}{v}\left(2 a_{0} a_{4}+2 a_{3} a_{1}+a_{2}^{2}\right)+4 a_{4}\right), \\
& a_{6}=\frac{-1}{132}\left(\frac{1}{v}\left(2 a_{0} a_{5}+2 a_{4} a_{1}+2 a_{3} a_{2}\right)+4 a_{5}\right) .
\end{aligned}
$$

Considering them as functions of $s$ all terms $a_{1}, \ldots, a_{6}$ are at most cubic. The conserve condition for $Q>0$ is

$$
\int_{-\frac{\theta_{0}}{2}}^{\frac{\theta_{0}}{2}} u(\theta) d \theta=Q
$$

This condition for the approximation $u(\theta) \approx \sum_{m=0}^{6} a_{m} \theta^{2 m}$ (which is a polynomial of degree 12 for $n=6$ ) is

$$
2 q a_{0}+\sum_{m=1}^{n} \frac{2 q^{2 m+1}}{2 m+1} a_{m}=Q
$$

where $q=\frac{\theta_{0}}{2}$, and is a cubic equation in $s$. In general for a fixed maximum value $E$, the $n$-Taylor's polynomial is a polynomial for $c$ and we can use numerical methods effectively to approximate $s$ such that the solution satisfies the conserve condition. 
Next we consider $x \in\left[-\frac{\pi}{4}, \frac{\pi}{4}\right]$ and study how the boundary values at $\pm \frac{\pi}{4}$ and $Q$ change by varying $E$ and $v$.

Experiment 1. Fix $v=1, Q=5$, then for $E=2, \ldots, 6$ from (42) with $n=9$ we estimate $s=[27.5,23.5,20.5,19,18.5]$. Next, using (35) we get the approximate solution shown in Figure 4.

The approximate values for $Q=[5.0656,5.0338,4.9880,4.9910,4.9874]$.

The solutions in Figure 4 show that the boundary conditions decrease smoothly to zero when $E$ varys from 2 to 5 , and become to negative for $E=6$. In the case $E>6$, the solution exists only for negative initial values. This might be considered corresponding to walls moving in opposite direction of the flow. Another observation is that for small values of $E$ but $Q=5$ the maximum velocity is along the walls.

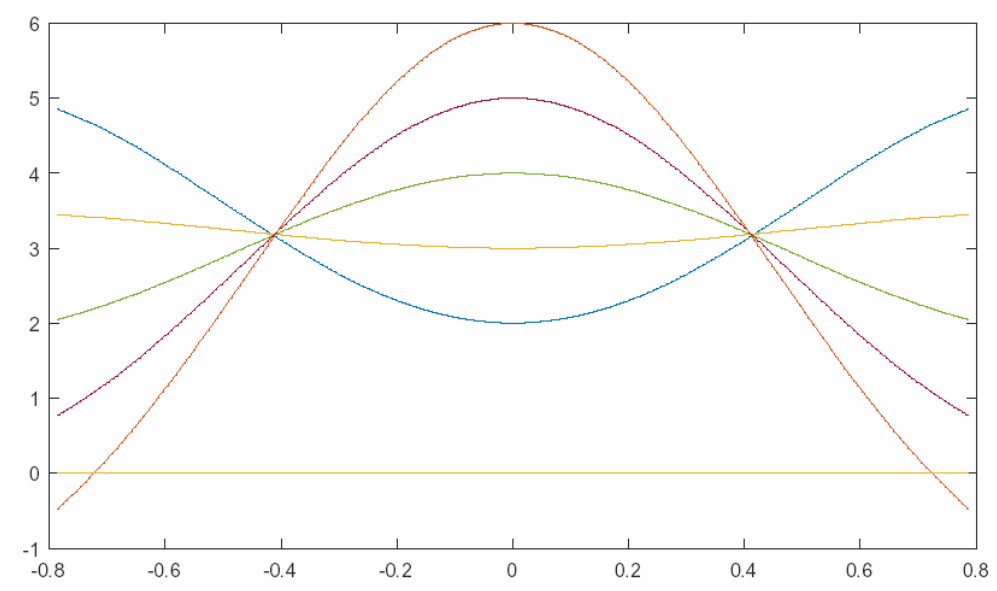

Figure 4. $Q=5$ and $E=2, \ldots, 6$.

Experiment 2. Fix $v=1, E=10$ and let $s$ from 52 to 86, skip 5. By using (35), we obtained the following solutions: 
The conserve quantity is $Q=[7.8194,8.3485,8.8662,9.3732,9.8700$, $10.3570,10.8347]$.

According to the numerical results shown in Figure 5, for a fixed maximum value, $E=10$, by varying $s$ the conserve quantity varies smoothly and the boundary conditions vary from negative to positive.

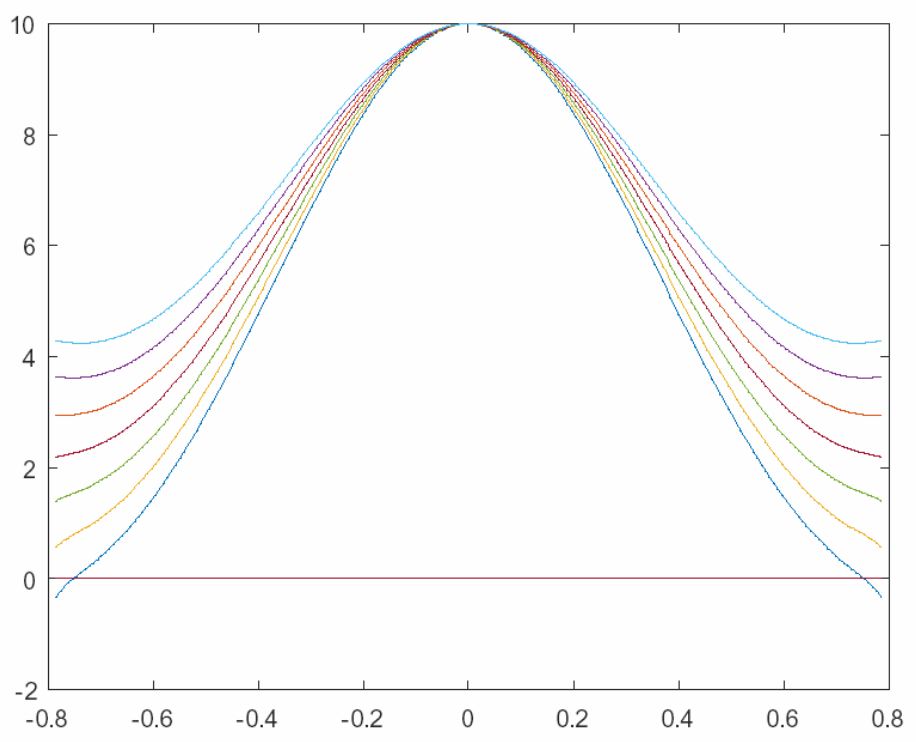

Figure 5. $E=10$ and $s$ varies from 52 to 86 , skip $s=5$.

Experiment 3. In the last experiment we study the effect when $v$ varies from 0.35 to 2.5 with a step 0.2 . The maximum value is fixed as $E=4$ and $s=17$. Using the McLauren series again we obtained the following solutions:

The conserve quantity is $Q=[2.3411,3.4708,4.0885,4.4909,4.7752$, $4.9873,5.1517,5.2830,5.3902,5.4795,5.5550]$. 
Figure 6 shows that for $v=0.35$ the initial conditions are negative and when $v$ increases they smoothly increase.

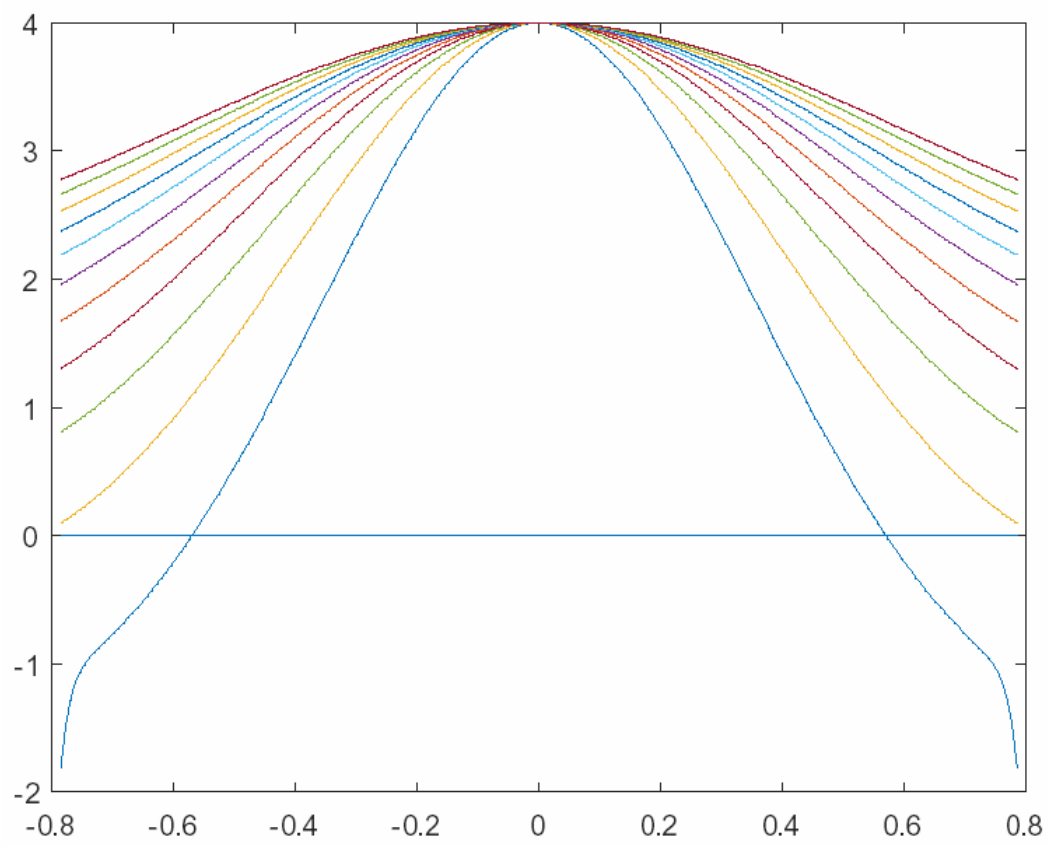

Figure 6. $E=10$ and $s$ varies from 52 to 86, skip $s=5$.

\section{Slip vs. No-Slip Boundary Conditions}

In this section, we show that the no-slip boundary condition can be obtained as an uniform limit of the slip boundary condition. Following the notation in (4) we introduce

$$
U_{s}=-\left.\lambda \mu \frac{d U}{d \theta}\right|_{\theta= \pm \frac{\theta_{0}}{2}},
$$


where $U_{s}$ is the fluid velocity along the wall $\theta= \pm \theta_{0} / 2$. For ease of notations we assume $\lambda=1$ and then the slip boundary condition (4) becomes $U_{s}=-\mu U_{s}^{\prime}$. When coupled with (33) it is equivalent to $U_{s}(\mu)$ being a positive solution of the cubic equation

$$
\frac{1}{\mu^{2}} U_{s}^{2}=f\left(U_{s}\right)
$$

where $f$ is introduced by (23).

The cubic polynomial $P(x, \mu)=\frac{2}{3 v} x^{3}+\left(4+\frac{1}{\mu}\right) x^{2}-2 c_{1} x+2 c_{2}$ as a function of $\mu$ is uniformly decreasing for any $x$, indeed

$$
\frac{\partial P}{\partial \mu}=-\frac{2}{\mu^{3}} x^{2}<0
$$

for $\mu>0$. When $\mu \rightarrow \infty, P(x, \mu) \approx x^{2}$ with one positive and one negative zeros in a neighbourhood of $x=0$, the third zero approaches $-\infty$. When $\mu \rightarrow \infty P(x, \mu) \rightarrow P(x, 0)$ and the zeros approach the zeros of $P(x, 0)$. Our interest is in the behaviour of $U_{s}(\mu)$ when $\mu \rightarrow 0$. From the above considerations, it follows that $U_{s}(\mu)$ is the greatest positive solution of the cubic equation $\mu P(x, \mu)=0$ and $U_{s}(0)=0$. By using the formulas for solutions of cubic equations (44), we can conclude that the positive zero $U_{s}(\mu)=0+O\left(\mu^{2}\right)$ when $\mu \rightarrow 0$. For any choice of $c_{1}, c_{2}$ and the power series expansion of $U$ it follows that $U(\theta, \mu) \rightarrow U(\theta, 0)$ as $\mu \rightarrow 0$. An example with viscosity $v=1$ and $c_{1}=35, c_{2}=17$ on the interval $\left[-\frac{\pi}{8}, \frac{\pi}{8}\right]$ is illustrated in Figure 6. Table 1 contains the values of $\mu$ and the corresponding $U_{s}(\mu)$ as well as the maximum velocity in each case. 
Table 1

\begin{tabular}{|c|c|c|c|c|c|c|c|c|c|c|c|c|}
\hline$\mu$ & 0 & 0.03 & 0.05 & 0.07 & 0.09 & 0.15 & 0.21 & 0.26 & 0.36 & 0.47 & 8 & 800 \\
\hline$U_{s}(\mu)$ & 0 & 0.20 & 0.37 & 0.54 & 0.88 & 1.81 & 2.85 & 3.73 & 4.42 & 4.42 & 4.42 & 4.42 \\
\hline$E$ & 17.50 & 17.25 & 17.00 & 16.75 & 16.25 & 14.75 & 12.75 & 10.50 & 6.25 & 6.25 & 6.25 & 6.25 \\
\hline
\end{tabular}

For small values of $\mu$ the boundary conditions are close to zero with maximum velocity in the velocity of 17 . For larger values of $\mu>0.36$ the solution $U$ convergence very fast to the limit case $U(\theta, 0)$. We see that slip boundary conditions result in solutions that uniformly converge to the solution in the no-slip case when $\mu \rightarrow 0$, which is also illustrated in Figure 7.
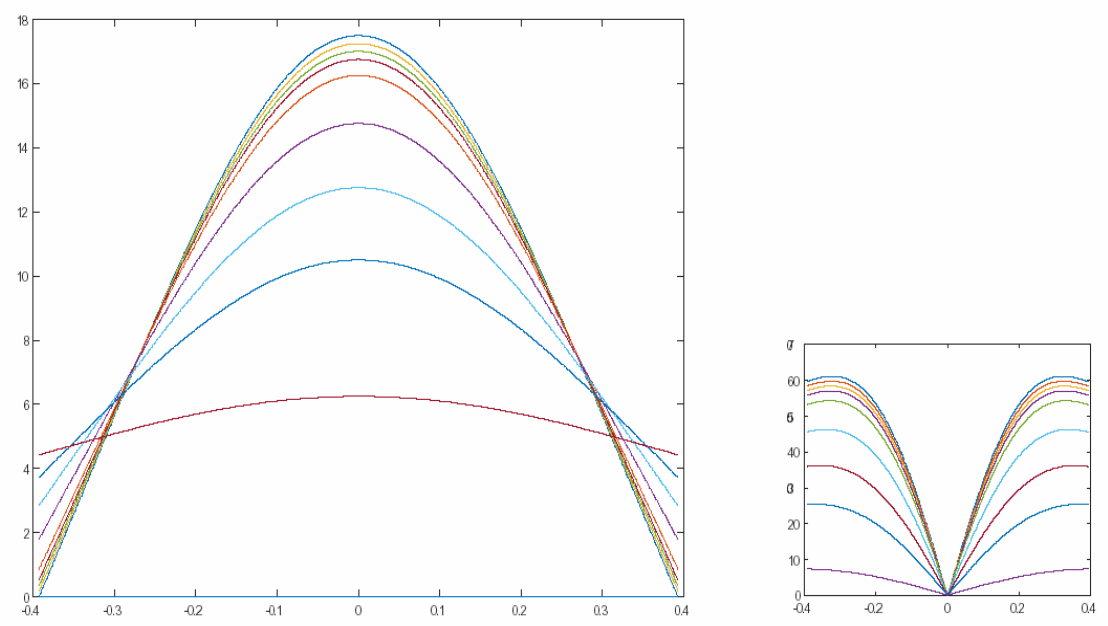

Figure 7. Slip vs no-slip BC: right graph is $U(\mu)$, left graph is $U_{\theta}(\mu)$.

\section{Conclusion}

We studied the correlation between no-slip and slip boundary conditions for Newtonian fluid for special case of a plane diffuser. The general solution of Equations (20) and (25) for the velocity is related to a half period shifted Weierstrass $P$ function with parameters $g_{2}, g_{3}$. The 
explicit solution obtained is a well known function in many areas of mathematics but for our analysis we provided a Taylor series expansion centered at 0 with two new parameters, the maximum velocity $E$ and the parameter $s$. In Section 7, we presented a numerical study of the flow described by two macro-parameters, the maximum flow and the conserved quantity $Q$. These two quantities can be easily measured in physical experiments. In a series of examples, we showed that the assumption for no-slip boundary conditions is stable in the sense that a small perturbation of the boundary values result in a small perturbation in the solutions.

In Section 8, we established that slip boundary conditions are uniform limit of no-slip boundary conditions and considered constructive method for solving the boundary value problem

$$
\begin{aligned}
\frac{d^{2} U}{d \theta^{2}}+4 U+\frac{1}{v} U^{2} & =c_{1} \\
U_{s} & =-\mu \frac{d U_{s}}{d \theta} .
\end{aligned}
$$

Since ejector-based systems provide a potentially promising solution, particularly for moderate heating or cooling applications, refrigeration and air conditioning, the results presented in this work might be useful in physical experiments studying the fluid velocity and with slip or no-slip effect. A better understanding the correlation between slip and no-slip boundary conditions in a diffuser may improve the technology of ejectorbased systems, which can recover low-grade energy available as waste heat in most industrial processes and use renewable energy or any other source at low cost.

\section{Acknowledgement}

We would like to thank the Dean of STEM \& Social Sciences at Wenatchee Valley College, Holly Bringman for providing us with all the necessary facilities for the research, great support, and encouragement. 


\section{References}

[1] Z. Aidoun, K. Ameur, M. Falsafioon and M. Badache, Current advances in ejector modeling, experimentation and applications for refrigeration and heat pumps, Part 1: Single-phase ejectors, Inventions 4(1) (2019); Article 15.

DOI: https://doi.org/10.3390/inventions4010015

[2] C. Appenzeller, C. H. Davies and W. A. Norton, Fragmentation of stratospheric intrusions, Journal of Geophysical Research 101(D1) (1996), 1435-1456.

DOI: https://doi.org/10.1029/95JD02674

[3] J. S. Carlton, in Marine Propellers and Propulsion, Fourth Edition, 2019.

[4] V. V. Chandra and M. R. Ahmed, Experimental and computational studies on a steam jet refrigeration system with constant area and variable area ejectors, Energy Conversion and Management 79 (2014), 377-386.

DOI: https://doi.org/10.1016/j.enconman.2013.12.035

[5] P. Desevaux, F. Lanzetta and Y. Bailly, CFD modelling of shock train inside a supersonic ejector: Validation against flow visualizations and pressure measurements in the case of zero-secondary flow, In Proceedings of the 10th International Symposium on Flow Visualization, Kyoto, Japan, 26-29 August, 2002.

[6] I. W. Eames, A. E. Ablwaifa and V. Petrenko, Results of an experimental study of an advanced jet-pump refrigerator operating with R245fa, Applied Thermal Engineering 27(17-18) (2007), 2833-2840.

DOI: https://doi.org/10.1016/j.applthermaleng.2006.12.009

[7] N. I. Akhiezer, Elements of the Theory of Elliptic Functions, Translations of Mathematical Monographs, Volume 79, American Mathematical Society, ISBN 0-8218-4532-2 (1990).

[8] J. Hawkins and L. Koss, Connectivity properties of Julia sets of Weierstrass elliptic functions, Topology and its Applications 152(1-2) (2005), 107-137.

DOI: https://doi.org/10.1016/j.topol.2004.08.018

[9] C. S. Iorio, O. Goncharova and O. Kabov, Influence of boundaries on shear-driven flow of liquids in open cavities, Microgravity Science and Technology 23(4) (2011), 373-379.

DOI: https://doi.org/10.1007/s12217-011-9257-6

[10] K. Ishizaka, K. Saitoh, E. Ito, M. Yuri and J. Masada, Key technologies for $1700^{\circ} \mathrm{C}$ class ultra high temperature gas turbine, Mitsubishi Heavy Industries Technical Review 54(3) (2017), 23-32. 
[11] T. S. Lundgren, P. R. Sethna and A. K. Bajaj, Stability boundaries for flow induced motions of tubes with an inclined terminal nozzle, Journal of Sound and Vibration 64(4) (1979), 553-571.

DOI: https://doi.org/10.1016/0022-460X(79)90804-6

[12] C. Neto, D. R. Evans, E. Bonaccurso, H.-J. Butt and V. S. J. Craig, Boundary slip in Newtonian liquids: A review of experimental studies, Reports on Progress in Physics 68(12) (2005), 2859-2897.

DOI: https://doi.org/10.1088/0034-4885/68/12/R05

[13] L. Prandtl, Zur berechnung der grenzschichten, ZAMM Journal of Applied Mathematics and Mechanics 18(1) (1938), 77-82.

DOI: https://doi.org/10.1002/zamm.19380180111

[14] P. N. Shankar and M. D. Deshpande, Fluid mechanics in the driven cavity, Annual Review of Fluid Mechanics 32 (2000), 93-136.

DOI: https://doi.org/10.1146/annurev.fluid.32.1.93

[15] H. A. Stone, A. D. Stroock and A. Ajdari, Engineering flows in small devices: Microfluidics toward a lab-on-a-chip, Annual Review of Fluid Mechanics 36 (2004), 381-411.

DOI: https://doi.org/10.1146/annurev.fluid.36.050802.122124

[16] H. Struchtrup and M. Torrilhon, Higher-order effects in rarefied channel flows, Physical Review E 78(4 Pt 2) (2008); Article 046301.

DOI: https://doi.org/10.1103/PhysRevE.78.046301

[17] P. Tabeling, Slip phenomena at liquid: Solid interfaces, Comptes Rendus Physique 5(5) (2004), 531-537.

DOI: https://doi.org/10.1016/j.crhy.2004.02.009

[18] L. Wang, W. Cai, H. Zhao, C. Lin and J. Yan, Experimentation and cycle performance prediction of hybrid A/C system using automobile exhaust waste heat, Applied Thermal Engineering 94 (2016), 314-323.

DOI: https://doi.org/10.1016/j.applthermaleng.2015.10.051

[19] R. Yapici, H. K. Ersoy, A. Aktoprakoglu, H. S. Halkaci and O. Yigit, Experimental determination of the optimum performance of ejector refrigeration system depending on ejector area ratio, International Journal of Refrigeration 31(7) (2008), 1183-1189.

DOI: https://doi.org/10.1016/j.ijrefrig.2008.02.010

[20] M. T. Zegenhagen and F. Ziegler, A one-dimensional model of a jet-ejector in critical double choking operation with R134a as a refrigerant including real gas effects, International Journal of Refrigeration 55 (2015), 72-84.

DOI: https://doi.org/10.1016/j.ijrefrig.2015.03.013 